\title{
Declining Mortality Rate of Hospitalised Patients in the Second Wave of the COVID-19 Epidemics in Italy: Risk Factors and the Age-Specific Patterns
}

\author{
Antonella D’Arminio Monforte *(D), Alessandro Tavelli, Francesca Bai, Daniele Tomasoni, Camilla Falcinella, \\ Roberto Castoldi, Diletta Barbanotti, Giovanni Mulè, Marina Allegrini, Elisa Suardi, Daniele Tesoro, \\ Gianmarco Tagliaferri (D, Debora Mondatore, Matteo Augello (D), Andrea Cona, Tomaso Beringheli, \\ Nicole Gemignani, Matteo Sala, Benedetta Varisco, Francesco Molà, Sofia Pettenuzzo, Lorenzo Biasioli, \\ Alessandro Copes, Lidia Gazzola, Ottavia Viganò, Camilla Tincati (D), Anna De Bona, Teresa Bini \\ and Giulia Marchetti
}

check for updates

Citation: D'Arminio Monforte, A.; Tavelli, A.; Bai, F.; Tomasoni, D.; Falcinella, C.; Castoldi, R.; Barbanotti, D.; Mulè, G.; Allegrini, M.; Suardi, E.; et al. Declining Mortality Rate of Hospitalised Patients in the Second Wave of the COVID-19 Epidemics in Italy: Risk Factors and the Age-Specific Patterns. Life 2021, 11, 979. https:// doi.org/10.3390/life11090979

Academic Editor: Matthias Clauss

Received: 6 August 2021

Accepted: 13 September 2021

Published: 17 September 2021

Publisher's Note: MDPI stays neutral with regard to jurisdictional claims in published maps and institutional affiliations.

Copyright: (c) 2021 by the authors. Licensee MDPI, Basel, Switzerland. This article is an open access article distributed under the terms and conditions of the Creative Commons Attribution (CC BY) license (https:// creativecommons.org/licenses/by/ $4.0 /)$.
Institute of Infectious Diseases, Department of Health Science, ASST Santi Paolo e Carlo, University of Milan, 20142 Milan, Italy; Alessandro.tavelli@gmail.com (A.T.); francesca.bai@unimi.it (F.B.);

daniele.tomasoni@unimi.it (D.T.); camilla.falcinella@unimi.it (C.F.); roberto.castoldi@unimi.it (R.C.); diletta.barbanotti@unimi.it (D.B.); giovanni.mule@unimi.it (G.M.); marina.allegrini@unimi.it (M.A.); elisa.suardi@asst-santipaolocarlo.it (E.S.); daniele.tesoro@unimi.it (D.T.); Gianmarco.tagliaferri@unimi.it (G.T.); Debora.mondatore@unimi.it (D.M.); matteo.augello@unimi.it (M.A.); andrea.cona@unimi.it (A.C.); Tomaso.beringheli@unimi.it (T.B.); nicole.gemignani@unimi.it (N.G.); Matteo.sala1@unimi.it (M.S.); benedetta.varisco@unimi.it (B.V.); francesco.mola@unimi.it (F.M.); sofia.pettenuzzo@unimi.it (S.P.); Lorenzo.biasioli@unimi.it (L.B.); alessandro.copes@unimi.it (A.C.); lidia.gazzola@asst-santipaolocarlo.it (L.G.); ottavia.vigano@asst-santipaolocarlo.it (O.V.); camilla.tincati@unimi.it (C.T.);

Anna.debona@asst-santipaolocarlo.it (A.D.B.); teresa.bini@unimi.it (T.B.); giulia.marchetti@unimi.it (G.M.)

* Correspondence: antonella.darminio@unimi.it; Tel.: +39-0281843045

Abstract: Background: Mortality rate from COVID-19 in Italy is among the world's highest. We aimed to ascertain whether there was any reduction of in-hospital mortality in patients hospitalised for COVID-19 in the second-wave period (October 2020-January 2021) compared to the first one (February-May 2020); further, we verified whether there were clusters of hospitalised patients who particularly benefitted from reduced mortality rate. Methods: Data collected related to in-patients' demographics, clinical, laboratory, therapies and outcome. Primary end-point was time to in-hospital death. Factors associated were evaluated by uni- and multivariable analyses. A flow diagram was created to determine the rate of in-hospital death according to individual and disease characteristics. Results: A total of 1561 patients were included. The 14-day cumulative incidence of in-hospital death by competing risk regression was of $24.8 \%$ (95\% CI: $21.3-28.5)$ and $15.9 \%$ (95\% CI: 13.7-18.2) in the first and second wave. We observed that the highest relative reduction of death from first to second wave (more than $47 \%$ ) occurred mainly in the clusters of patients younger than 70 years. Conclusions: Progress in care and supporting therapies did affect population over 70 years to a lesser extent. Preventive and vaccination campaigns should focus on individuals whose risk of death from COVID-19 remains high.

Keywords: COVID-19; hospitalised patients; age; in-hospital mortality rate; first and second wave of epidemics

\section{Introduction}

In the last 2-year period SARS CoV-2 pandemics has caused over 4.2 million deaths worldwide [1], causing major socio-economic and health disruptions worldwide. The pandemics has spread all over Europe at different waves, the first one occurring in MarchMay 2020, the second one in October-January 2021 and the third one in March-May 2021. Finally, a fourth wave including highly contagious virus variants is ongoing all over the 
world. While the first wave was unexpected and resulted in sudden dramatic changes to health system in different countries, the second one was foreseeable and hospitals were already organised to host a number of COVID-19 in ordinary, subacute and ICU beds.

Mortality rates vary widely according to different settings and in different parts of the world [2,3]. This high variability might depend on different factors, first of all the reference population, including either only hospitalised or also out-patients, but also the health setting including the availability of ICU beds, and individual variables, such as socioeconomical status [3]; all these factors might contribute to disentangle differences on COVID-19 fatality rates in different settings.

In this context, Italy was the first European country for number of deaths well above the number of 100,000 [4]. Importantly, most deaths were in Lombardy, accounting for $49 \%$ of the country's total [5]. Given this unexplained high frequency in this region, we aimed at understanding any possible reasons behind such trend.

The aims of this study are to ascertain any differences in in-hospital mortality rates according to the two waves periods, and to verify whether the contribution of patients characteristics and disease presentation to the outcome has a different weight in the first and second waves.

\section{Materials and Methods}

The prospective observational cohort includes all $\geq 18$ years old patients hospitalised for confirmed SARS CoV-2 symptomatic infection (positive RT-PCR from nasopharyngeal or broncho-alveolar swab) from 24 February 2020 to 31 January 2021 at San Paolo University hospital, in Milan. Patients who died in emergency room within $24 \mathrm{~h}$ and patients not hospitalised were not included.

We divided the whole period into three groups: from 24 February to 31 May 2020 (first period), from 1 June to 30 September 2020 (second period) and from 1 October 2020 to 31 January 2021 (third period).

Data were entered into an electronic database, including: demographics; risk factors for SARS CoV-2; comorbidities; age-unadjusted Charlson comorbidity index [6]; symptoms; laboratory examinations at admission. Disease severity at admission was classified as mild (no pneumonia); moderate (pneumonia by X-ray; $\mathrm{RR}>26 / \mathrm{min} ; \mathrm{SO}_{2}>96 \%$ in room air; $\mathrm{PaO}_{2} / \mathrm{FiO}_{2}>300 \mathrm{mmHg}$ ); severe ( $\mathrm{RR}<24 / \mathrm{min} ; \mathrm{SO}_{2}<92 \% ; \mathrm{PaO}_{2} / \mathrm{FiO}_{2} 100-300 \mathrm{mmHg}$ ); critical disease $\left(\mathrm{PaO}_{2} / \mathrm{FiO}_{2}<100 \mathrm{mmHg}\right)$.

The highest intensity of ventilation during hospitalisation was recorded as: no need; low/high flow supplemental oxygen by nasal cannula/face mask; continuous positive airway pressure device (cPAP); mechanical non-invasive (NIV); or invasive ventilation (IV).

Use of remdesivir or other antivirals, immunomodulatory agents (tocilizumab, sarilumab, ruxolitinib, baricitinib) and high-dose corticosteroids (dexamethasone and methylprednisolone) were also collected. Primary endpoint was time to in-hospital death, discharge from hospital was analysed as a competing event by competing risks analysis.

Continuous variables are presented as median and interquartile ranges (IQR), categorical variables are presented as frequency and percentage. Difference according to calendar period of admission are evaluated by Kruskal-Wallis and Chi-square tests, as appropriate.

In-hospital mortality has been evaluated by competing risks analysis, using cumulative incidence function (CIF). In our study, discharge from hospital after recovery is considered a competing risk for in-hospital mortality, whereas in standard survival analyses (i.e., Kaplan Meier curves), patients who recover are censored. This censoring violates the assumption of noninformative censoring, as the recovered and discharge patients are not representative of those who are still admitted to the hospital in terms of their risk of dying. Censoring patients induce bias and overestimate the incidence of death.

A proportional sub-distribution hazard (SHR) model by Fine and Gray has been fitted to estimate the effect of calendar period and other covariates on CIFs in-hospital death. Pepe-Mori test was used to compare equality of CIFs across subgroups (waves of 
epidemic). A sub-group analysis has been performed using the subpopulation of patients severe/critical at hospital admission.

Two different sets of covariates, chosen a priori, has been used in the adjusted analyses, Model 1 does not include data on therapy used for COVID-19 while Model 2 includes as covariates the anti-COVID19 regimen used (remdesivir, corticosteroids, immunomodulators). Actually, both remdesivir and desamethasone were demonstrated to be effective after the first wave, on May 2020 for remdesivir and on July 2020 for desamethasone. Thereafter, they became the regimen of choice. Of note, remdesivir should be used only within 10 days from the onset of symptoms.

We have also investigated the in-hospital mortality by age strata ( $<70$ and $\geq 70$ years old).

Moreover, we have classified the participants in 16 sub-groups/clusters according to age ( $<70$ and $\geq 70$ years), sex (male and female), COVID-19 severity at admission (mild/moderate vs. severe/critical) and burden of comorbidities (age-unadjusted CCI $<2$ and $\geq 2$ ). We then calculated the marginal probabilities of in-hospital death by fitting a logistic regression model with age, sex, age-unadjusted CCI, disease severity, LDH, lymphocytes count, CRP, D-dimer, and obesity as covariates without interactions and estimated the probability of in-hospital death in two calendar periods (February/May 2020 and October-2020/January-2021) according to the 16 participant's clusters. The predicted probabilities of in-hospital death by calendar period for each cluster has been plotted to evaluate the overall and relative changes.

All the statistical analyses have been performed using Stata (version 14.0, StataCorp, College Station, TX, USA).

\section{Results}

A total of 1683 patients were hospitalised for COVID-19 at San Paolo Hospital from 24 February to 31 January 2021: 556 (33.0\%) between February and May (first wave), 1005 (59.7\%) between October 2020 and January 2021 (second wave), and $122(7.2 \%)$ in the interweaves period (June-September); these last patients were not included in the analyses, due to the low number and different characteristics (Supplementary Table S1). Demographic and clinical characteristics of the remaining 1561 patients are reported in Table 1; differences in patients' and clinical characteristics were examined according to the two calendar periods (waves of epidemics). Second-wave patients were older (second vs. first wave: median age 72 years-IQR 57-81 vs. 66 years-IQR 55-78, $p<001$ ), suffering from more comorbidities (median age-unadjusted Charlson index second vs. first wave: 1 point-IQR $0-2$ vs. 0 pointsIQR 0-2. $p=0.003$ ), and displayed a less severe SARS CoV-2-related disease (no pneumonia: $11.6 \%$ vs. $5.6 \% ; p<001$ ). A number of disease-related symptoms and signs were different according to the different calendar periods. Noteworthy, in the second calendar period patients were referred to hospital earlier, a median of 5 days (IQR: 3-8) vs. 7 days (IQR: 3.10) from the beginning of symptoms. According to new evidence from trials remdesivir, corticosteroids and biological agents were all used more frequently in the second-wave patients. In-hospital death occurred in $178(32 \%)$ patients belonging to the first wave and $245(24.4 \%)$ patients belonging to the second-one $(p<001)$ (see Table 1$)$.

The 14-day cumulative incidence of in-hospital death by competing risk regression was of $24.8 \%$ (95\% CI: $21.3-28.5)$ and $15.9 \%$ (95\% CI: 13.7-18.2) in the first and second wave, respectively (pepe-mori test $p$ : <001).

Looking to possible factors associated to in-hospital death by multivariable models, both considering in the model the use of drugs or not, patients belonging to the second wave showed a significant reduction of risk as compared to the first-wave patients (model 1 not considering drugs: ASHR 0.59. 95\% CI: 0.48-0.74; model 2 including drugs: ASHR 0.61-95\% CI: 0.47-0.78). Other independent variables associated with higher risk of in-hospital death were age (Model1: ASHR: 1.66-95\% CI: 1.52-1.82), male sex (ASHR: 1.24-95\% CI: 1.00-1.54). Diseases related parameters, such as Charlson Index, proinflammatory markers and disease severity at admission were all associated with the risk of in-hospital death (Table 2). 
Table 1. Demographic, clinical and laboratory characteristics and outcomes of 1561 patients according to the period of SARS CoV-2 epidemics.

\begin{tabular}{|c|c|c|c|c|c|c|c|}
\hline \multirow[b]{3}{*}{ Age, years, median (IQR) } & \multirow{2}{*}{\multicolumn{2}{|c|}{$\begin{array}{c}\text { February 2020-June } 2020 \\
\qquad N=556(35.6)\end{array}$}} & \multirow{2}{*}{\multicolumn{2}{|c|}{$\begin{array}{c}\text { October 2020-January } 2021 \\
\qquad N=1005(64.4)\end{array}$}} & \multicolumn{2}{|c|}{ Total } & \multirow{3}{*}{$\begin{array}{c}p \\
0.001\end{array}$} \\
\hline & & & & & \multicolumn{2}{|c|}{$N=1561(100.0)$} & \\
\hline & 66 & $55-8$ & 72 & $57-81$ & 70 & $56-81$ & \\
\hline Age, $>70$ years, $\mathrm{n}(\%)$ & 249 & 44.8 & 547 & 54.0 & 796 & 51.0 & $<0.001$ \\
\hline Sex, Male & 356 & 64.0 & 635 & 63.2 & 991 & 63.5 & 0.740 \\
\hline Italian & 442 & 79.5 & 808 & 81.2 & 1250 & 80.6 & 0.414 \\
\hline Ethnicity & & & & & & & 0.044 \\
\hline Caucasian & 460 & 82.7 & 836 & 83.9 & 1296 & 83.5 & \\
\hline Latin/Hispanic & 45 & 8.1 & 60 & 6 & 105 & 6.8 & \\
\hline Black & 8 & 1.44 & 4 & 0.4 & 12 & 0.8 & \\
\hline Asian & 16 & 2.9 & 45 & 4.5 & 61 & 3.9 & \\
\hline Other & 27 & 4.9 & 52 & 5.2 & 79 & 5.1 & \\
\hline Epidemiology, n (\%) & & & & & & & $<0.001$ \\
\hline Close contact & 84 & 15.1 & 50 & 5 & 134 & 8.6 & \\
\hline Healthcare workers & 43 & 7.7 & 10 & 1 & 53 & 3.4 & \\
\hline Hospitalisation & 31 & 5.6 & 171 & 17 & 202 & 12.9 & \\
\hline Long-term care facility & 64 & 11.5 & 48 & 4.8 & 112 & 7.2 & \\
\hline Other/Unknown & 334 & 60.1 & 726 & 72.2 & 1060 & 67.9 & \\
\hline Smoking, n (\%) & & & & & & & $<0.001$ \\
\hline Never smoker & 54 & 9.7 & 33 & 3.3 & 87 & 5.6 & \\
\hline Former smoker & 57 & 10.3 & 72 & 7.1 & 57 & 72 & \\
\hline Actual smoker & 13 & 2.3 & 47 & 4.7 & 60 & 3.8 & \\
\hline Unknown & 432 & 77.7 & 853 & 84.9 & 1285 & 82.3 & \\
\hline Obesity, n (\%) & & & & & & & $<0.001$ \\
\hline No & 206 & 37.1 & 139 & 13.8 & 345 & 22.1 & \\
\hline Yes & 89 & 16 & 107 & 10.7 & 196 & 12.6 & \\
\hline Unknown & 261 & 46.9 & 759 & 75.5 & 1020 & 65.3 & \\
\hline BMI, $\mathrm{kg} / \mathrm{m}^{2}$, median (IQR) & 27.4 & $23.9-31.5$ & 26.2 & $23.5-30.7$ & 26.7 & $23.7-31.2$ & 0.194 \\
\hline Hypertension, $\mathrm{n}(\%)$ & 260 & 46.8 & 514 & 51.1 & 774 & 49.6 & 0.097 \\
\hline Stroke, n (\%) & 49 & 8.8 & 99 & 9.9 & 148 & 9.5 & 0.503 \\
\hline CPD, n (\%) & 48 & 8.6 & 97 & 9.7 & 145 & 9.3 & 0.507 \\
\hline IMA, n (\%) & 72 & 13 & 175 & 17.4 & 247 & 15.8 & 0.021 \\
\hline Diabetes, $\mathbf{n}(\%)$ & 100 & 18 & 224 & 22.3 & 324 & 20.8 & 0.045 \\
\hline Cerebrovascular diseases, $\mathrm{n}(\%)$ & 49 & 8.8 & 99 & 9.9 & 148 & 9.5 & 0.503 \\
\hline Cardiovascular diseases $\mathrm{n}(\%)$ & 154 & 27.7 & 341 & 33.9 & 495 & 31.7 & 0.011 \\
\hline COPD/Asthma, n (\%) & 79 & 14.2 & 137 & 13.6 & 216 & 13.8 & 0.752 \\
\hline Cancer (last 5 years), n (\%) & 39 & 7 & 88 & 8.8 & 127 & 8.1 & 0.228 \\
\hline CKD, n (\%) & 44 & 7.9 & 84 & 8.4 & 128 & 8.2 & 0.759 \\
\hline Rheumatological Diseases, n (\%) & 15 & 2.7 & 12 & 1.2 & 27 & 1.7 & 0.029 \\
\hline Peripheric vascular diseases, $\mathrm{n}(\%)$ & 53 & 9.5 & 63 & 6.3 & 116 & 7.4 & 0.019 \\
\hline HIV, n (\%) & 4 & 0.7 & 11 & 1.1 & 15 & 1 & 0.467 \\
\hline Chronic liver disease, n (\%) & 20 & 3.6 & 44 & 4.9 & 64 & 4.1 & 0.456 \\
\hline $\begin{array}{c}\text { Age Unadjusted Charlson score, } \\
\text { median (IQR) }\end{array}$ & 0 & $0-2$ & 1 & $0-2$ & 1 & $0-2$ & 0.003 \\
\hline $\begin{array}{c}\text { Age Adjusted Charlson score, } \\
\text { median (IQR) }\end{array}$ & 3 & $1-5$ & 4 & $2-5$ & 3 & $1-5$ & $<0.001$ \\
\hline \multicolumn{8}{|l|}{ Signs and symptoms at admission } \\
\hline Fever & 475 & 85.4 & 698 & 69.5 & 1173 & 75.1 & $<0.001$ \\
\hline Dyspnoea & 308 & 55.4 & 492 & 49 & 800 & 51.3 & 0.015 \\
\hline Cough & 276 & 50 & 332 & 33 & 608 & 39 & $<0.001$ \\
\hline Fatigue & 89 & 16 & 150 & 14.9 & 239 & 15.3 & 0.570 \\
\hline GI Symptoms & 80 & 14.4 & 115 & 11.4 & 195 & 12.5 & $<0.001$ \\
\hline Erythromelalgia & 29 & 5.2 & 45 & 4.5 & 74 & 4.7 & 0.511 \\
\hline Chest pain & 26 & 4.7 & 36 & 3.6 & 62 & 4 & 0.289 \\
\hline Anosmia/dysgeusia & 18 & 3.2 & 57 & 5.67 & 75 & 4.8 & 0.031 \\
\hline Syncope/Pre-syncope & 10 & 1.8 & 48 & 4.8 & 58 & 3.72 & 0.003 \\
\hline
\end{tabular}


Table 1. Cont.

\begin{tabular}{|c|c|c|c|c|c|c|c|}
\hline & \multicolumn{2}{|c|}{ February 2020-June 2020} & \multicolumn{2}{|c|}{ October 2020-January 2021} & \multicolumn{2}{|c|}{ Total } & \multirow[t]{2}{*}{$p$} \\
\hline & \multicolumn{2}{|c|}{$N=556(35.6)$} & \multicolumn{2}{|c|}{$N=1005(64.4)$} & \multicolumn{2}{|c|}{$N=1561(100.0)$} & \\
\hline & & & & & & & \\
\hline No pneumonia & 31 & 5.6 & 117 & 11.6 & 148 & 9.5 & \\
\hline Mild & 254 & 45.7 & 394 & 39.2 & 648 & 41.5 & \\
\hline Severe & 253 & 45.5 & 468 & 46.6 & 721 & 46.2 & \\
\hline Critical & 18 & 3.2 & 26 & 2.6 & 44 & 2.8 & \\
\hline $\begin{array}{c}\text { Respiratory rate at admission, } \\
\text { breaths/min, median (IQR) }\end{array}$ & 24 & $20-29$ & 20 & $18-24$ & 22 & $18-26$ & $<0.001$ \\
\hline $\begin{array}{c}\text { X-ray or CT scan signs of pneumonia } \\
\text { Highest grade of } \mathrm{O}_{2}\end{array}$ & 514 & 92.4 & 804 & 80 & 1318 & 84.4 & $<0.001$ \\
\hline $\begin{array}{c}\text { therapy/ventilation during } \\
\text { hospitalisation }\end{array}$ & & & & & & & $<0.001$ \\
\hline Invasive mechanical ventilation (IMV) & 69 & 12.4 & 32 & 3.2 & 101 & 6.5 & \\
\hline $\begin{array}{c}\text { Non-invasive mechanical } \\
\text { Ventilation (NIV) }\end{array}$ & 52 & 9.4 & 63 & 6.3 & 115 & 7.4 & \\
\hline $\begin{array}{l}\text { Continuous positive airway } \\
\text { pressure (CPAP) }\end{array}$ & 157 & 28.2 & 273 & 27.2 & 430 & 27.6 & \\
\hline $\mathrm{O}_{2}$ low/high flows & 207 & 37.2 & 479 & 47.7 & 686 & 44 & \\
\hline $\mathrm{No} \mathrm{O}_{2}$ therapy & 71 & 12.8 & 158 & 15.7 & 229 & 14.7 & \\
\hline ICU admission, $\mathbf{n}(\%)$ & 72 & 13 & 38 & 3.8 & 110 & 7.1 & $<0.001$ \\
\hline \multicolumn{8}{|l|}{ Laboratory parameters } \\
\hline Haemoglobin/dL, median (IQR) & 13.5 & $12.1-14.8$ & 13.3 & $11.7-14.5$ & 13.3 & $11.8-14.6$ & 0.027 \\
\hline $\mathrm{CRP}, \mathrm{mg} / \mathrm{L}$, median $(\mathrm{IQR})$ & 60 & $26.8-102.2$ & 53.4 & $22.3-92.9$ & 54.9 & $24-95.6$ & $<0.001$ \\
\hline LDH, U/L, median (IQR) & 296 & 229-393 & 290 & $231-385$ & 292 & $230-389$ & 0.614 \\
\hline $\begin{array}{c}\text { Leukocytes count, } 10^{3} / \mathrm{uL} \\
\text { median }(\mathrm{IQR})\end{array}$ & 6.58 & $4.93-9.18$ & 7.31 & $5.26-10.11$ & 7 & $5.13-9.69$ & 0.002 \\
\hline $\begin{array}{l}\text { Lymphocyte count, } 10^{3} / \mathrm{uL} \text {, } \\
\text { median (IQR) }\end{array}$ & 1.02 & $0.68-1.37$ & 0.98 & $0.69-1.4$ & 0.99 & $0.68-1.38$ & 0.719 \\
\hline $\begin{array}{c}\text { Neutrophil count, } 10^{3} / \mathrm{uL} \\
\text { median }(\mathrm{IQR})\end{array}$ & 4.76 & $3.35-7.44$ & 5.34 & $3.64-8.12$ & 3.15 & $3.53-7.85$ & 0.002 \\
\hline Monocyte count, $10^{3} / \mathrm{uL}$, median (IQR) & 0.46 & $0.31-0.65$ & 0.5 & $0.34-0.73$ & 0.49 & $0.33-0.71$ & 0.004 \\
\hline Platlets, $10^{3} / \mathrm{uL}$, median (IQR) & 204 & $158-263$ & 208 & $161-266$ & 206 & $160-265$ & 0.443 \\
\hline $\begin{array}{l}\text { Creatine phosphokinase, } \mathrm{U} / \mathrm{L}, \\
\text { median (IQR) }\end{array}$ & 94 & 53-185 & 82 & 51-159 & 86 & $52-166$ & 0.052 \\
\hline D-Dimer,ng/mL, median (IQR) & 413 & $247-865$ & 350 & $218-660$ & 364 & $226-724$ & 0.002 \\
\hline ALT, U/L, median (IQR) & 29 & $20-49$ & 26 & $18-44$ & 27 & $19-45$ & 0.009 \\
\hline AST, U/L, median (IQR) & 41 & $30.5-60$ & 39 & $29-56$ & 40 & $30-57$ & 0.074 \\
\hline Creatinine, mg/dL, median (IQR) & 0.9 & $0.7-1.2$ & 0.9 & $0.7-1.2$ & 0.9 & $0.7-1.2$ & \\
\hline Procalcitonin, ng/mL, median (IQR) & 0.18 & $0.07-0.85$ & 0.15 & $0.05-0.45$ & 0.16 & $0.05-0.56$ & 0.031 \\
\hline Ferritin, ng/mL, median (IQR) & 447 & $219-858$ & 392 & 164-905 & 419 & $180-872$ & 0.119 \\
\hline $\begin{array}{l}\text { Days from symptoms onset and } \\
\text { hospitalisation, median (IQR) } \\
\text { Pharmacological treatments }\end{array}$ & 7 & $3-10$ & 5 & $2-7$ & 5 & $3-8$ & $<0.001$ \\
\hline Azithromycin, $\mathrm{n}(\%)$ & 168 & 30.2 & 25 & 2.5 & 193 & 12.4 & $<0.001$ \\
\hline Lopinavir/r or Darunavir/c, n (\%) & 133 & 23.9 & 0 & 0 & 133 & 8.5 & $<0.001$ \\
\hline Hydroxychloroquine, n (\%) & 434 & 78.1 & 1 & 0.1 & 435 & 27.9 & $<0.001$ \\
\hline Remdesivir, $\mathrm{n}(\%)$ & 9 & 1.6 & 206 & 20.5 & 215 & 13.8 & $<0.001$ \\
\hline Heparin prophylaxis, n (\%) & 360 & 64.8 & 784 & 78 & 1144 & 73.3 & $<0.001$ \\
\hline Corticosteroid treatment, $\mathrm{n}(\%)$ & 127 & 22.8 & 719 & 71.5 & 846 & 54.2 & $<0.001$ \\
\hline $\begin{array}{c}\text { Biological (Tocilizumab, Sarilumab), } \\
\mathrm{n}(\%)\end{array}$ & 44 & 7.9 & 29 & 2.9 & 73 & 4.7 & $<0.001$ \\
\hline $\begin{array}{c}\text { Days of Hospitalisation, median } \\
\text { (IQR) }\end{array}$ & 10 & $6-21$ & 10 & 6-19 & 10 & $6-20$ & 0.304 \\
\hline In-hospital death, n (\%) & 178 & 32 & 245 & 24.4 & 423 & 27.1 & $<0.001$ \\
\hline
\end{tabular}


Table 2. Factors associated with in-hospital mortality in 1561 patients hospitalised for COVID-19.

\begin{tabular}{|c|c|c|c|c|c|c|c|c|c|c|c|c|}
\hline \multirow[b]{3}{*}{ Age, per 10 years older } & \multicolumn{4}{|c|}{ Unadjusted } & \multicolumn{4}{|c|}{ Model1 (Without Drugs) } & \multicolumn{4}{|c|}{ Model2 (With Drugs) } \\
\hline & \multirow{2}{*}{$\begin{array}{c}\text { SHR } \\
1.73\end{array}$} & \multicolumn{2}{|c|}{$95 \% \mathrm{CI}$} & \multirow{2}{*}{$\begin{array}{c}p \\
<0.001\end{array}$} & \multirow{2}{*}{$\begin{array}{c}\text { aSHR } \\
1.66\end{array}$} & \multicolumn{2}{|c|}{$95 \%$ CI } & \multirow{2}{*}{$\begin{array}{c}p \\
<0.001\end{array}$} & \multirow{2}{*}{$\begin{array}{c}\text { aSHR } \\
1.67\end{array}$} & \multicolumn{2}{|c|}{$95 \% \mathrm{CI}$} & \multirow{2}{*}{$\begin{array}{c}p \\
<0.001\end{array}$} \\
\hline & & 1.61 & 1.85 & & & 1.52 & 1.82 & & & 1.52 & 1.83 & \\
\hline Sex, male (vs. female) & 1.08 & 0.89 & 1.32 & 0.438 & 1.24 & 1.00 & 1.54 & 0.045 & 1.26 & 1.01 & 1.56 & 0.040 \\
\hline \multicolumn{13}{|l|}{ Obesity $\left(B M I>30 \mathrm{~kg} / \mathrm{m}^{2}\right)$} \\
\hline No & 1.00 & & & & 1.00 & & & & 1.00 & & & \\
\hline Yes & 1.39 & 0.98 & 1.96 & 0.064 & 1.50 & 1.04 & 2.15 & 0.029 & 1.47 & 1.02 & 2.11 & 0.038 \\
\hline Unknown & 1.44 & 1.12 & 1.86 & 0.005 & 1.33 & 1.01 & 1.74 & 0.040 & 1.30 & 0.99 & 1.70 & 0.060 \\
\hline \multicolumn{13}{|l|}{$\begin{array}{l}\text { Charlson age unadjusted } \\
\text { index }\end{array}$} \\
\hline 0 & 1.00 & & & & 1.00 & & & & 1.00 & & & \\
\hline 1 & 2.61 & 2.00 & 3.42 & $<0.001$ & 1.83 & 1.39 & 2.42 & $<0.001$ & 1.85 & 1.40 & 2.44 & $<0.001$ \\
\hline 2 & 2.79 & 2.05 & 3.80 & $<0.001$ & 1.79 & 1.28 & 2.48 & 0.001 & 1.79 & 1.29 & 2.49 & 0.001 \\
\hline$\geq 3$ & 4.50 & 3.48 & 5.81 & $<0.001$ & 2.35 & 1.76 & 3.12 & $<0.001$ & 2.30 & 1.72 & 3.07 & $<0.001$ \\
\hline $\mathrm{LDH}>300 \mathrm{U} / \mathrm{L}(\mathrm{vs} \leq 300)$ & 1.99 & 1.62 & 2.45 & $<0.001$ & 1.52 & 1.21 & 1.91 & $<0.001$ & 1.51 & 1.20 & 1.90 & $<0.001$ \\
\hline $\begin{array}{c}\text { Lymphocyte }<1.0010^{3} / \mathrm{uL} \\
(\text { vs } \geq 1.000)\end{array}$ & 1.95 & 1.60 & 2.39 & $<0.001$ & 1.27 & 1.03 & 1.66 & 0.027 & 1.29 & 1.03 & 1.61 & 0.024 \\
\hline $\mathrm{CRP}>60 \overline{\mathrm{mg}} / \mathrm{L}(\mathrm{vs} \leq 60)$ & 2.80 & 2.29 & 3.43 & $<0.001$ & 1.80 & 1.44 & 2.25 & $<0.001$ & 1.80 & 1.43 & 2.25 & $<0.001$ \\
\hline $\begin{array}{c}\text { D-dimer }>1.000 \mathrm{ng} / \mathrm{mL} \\
\text { (vs. } \leq 1.000)\end{array}$ & 2.27 & 1.80 & 2.85 & $<0.001$ & 1.28 & 1.00 & 1.65 & 0.049 & 1.28 & 1.00 & 1.64 & 0.053 \\
\hline Severity & & & & & & & & & & & & \\
\hline mild/moderate & 1.00 & & & & 1.00 & & & & 1.00 & & & \\
\hline severe & 2.75 & 2.23 & 3.39 & $<0.001$ & 1.96 & 1.56 & 2.46 & $<0.001$ & 1.98 & 1.57 & 2.50 & $<0.001$ \\
\hline critical & 9.09 & 6.07 & 13.60 & $<0.001$ & 5.19 & 3.43 & 7.86 & $<0.001$ & 5.18 & 3.45 & 7.77 & $<0.001$ \\
\hline \multicolumn{13}{|l|}{ anti-COVID-19 regimen } \\
\hline None & 1.00 & & & & & & & & 1.00 & & & \\
\hline Immunomodulators only & 1.0481 & 0.53 & 2.07 & 0.892 & & & & & 0.66 & 0.30 & 1.49 & 0.320 \\
\hline $\begin{array}{c}\text { Immunomodulators + } \\
\text { Corticosteroids + Remdesivir }\end{array}$ & 0.5717 & 0.16 & 2.04 & 0.388 & & & & & 0.88 & 0.24 & 3.18 & 0.841 \\
\hline $\begin{array}{l}\text { Immunomodulators }+ \\
\text { Corticosteroids }\end{array}$ & 1.3293 & 0.75 & 2.35 & 0.327 & & & & & 1.24 & 0.66 & 2.32 & 0.505 \\
\hline Remdesivir & 0.4228 & 0.14 & 1.28 & 0.127 & & & & & 0.47 & 0.15 & 1.48 & 0.197 \\
\hline Corticosteroids & 1.0591 & 0.86 & 1.30 & 0.586 & & & & & 0.98 & 0.76 & 1.28 & 0.902 \\
\hline $\begin{array}{c}\text { Corticosteroids }+ \text { Remdesivir } \\
\text { Waves }\end{array}$ & 0.6977 & 0.50 & 0.98 & 0.036 & & & & & 0.78 & 0.53 & 1.14 & 0.195 \\
\hline March 2020-May 2020 & 1.00 & & & & 1.00 & & & & 1.00 & & & \\
\hline October 2020-January 2021 & 0.71 & 0.58 & 0.86 & $<0.001$ & 0.59 & 0.48 & 0.74 & $<0.001$ & 0.61 & 0.47 & 0.78 & $<0.001$ \\
\hline
\end{tabular}

Data were confirmed in the subgroup of patients with critical disease at admission; in these patients the association remdesivir+desamethasone was associated with a significant reduction of risk of in-hospital death (ASHR: 0.53-95\% CI: $0.34-0.82 ; p=005$ ) (Supplementary Table S2).

To address the second aim of our study, we explored whether the reduction in inhospital death in the second-wave patients was obtained mainly in selected clusters of patients. We therefore grouped the patients according to several characteristics: sex (M vs. F), age ( $\geq 70$ vs. $<70$ ), Charlson $\geq 2$ vs. $<2$ ), COVID- 19 severity at presentation (mild/moderate vs. severe/critical) and obtained 16 different groups; we then compared the differences of in-hospital deaths (second vs. first wave) accordingly.

In all cases there was a reduction in the predictive probability of death in the second wave as compared to the first one, but it is impressive to note that patients younger than 70 benefitted with more than $45 \%$ of relative reduction, whereas patients older than 70 showed always less than $30 \%$ reduction of in-hospital death. Inside these two groups, there was a gradient of benefit according to Charlson, sex and severity of COVID-19 at presentation; being man, older than 70 , with Charlson $<2$ and mild/moderate disease, the group with less benefit (17.6\% of in-hospital death reduction) and younger than 70, females, with Charlson $<2$ and severe/critical disease, the group benefiting more (in-hospital death reduction 
by $58 \%$ ). Data on reduction of predicted probability of in-hospital death in the second compared to the first wave, and of the overall predicted probability death in the individual groups according to the period are shown in Figure 1 (left and right part, respectively).

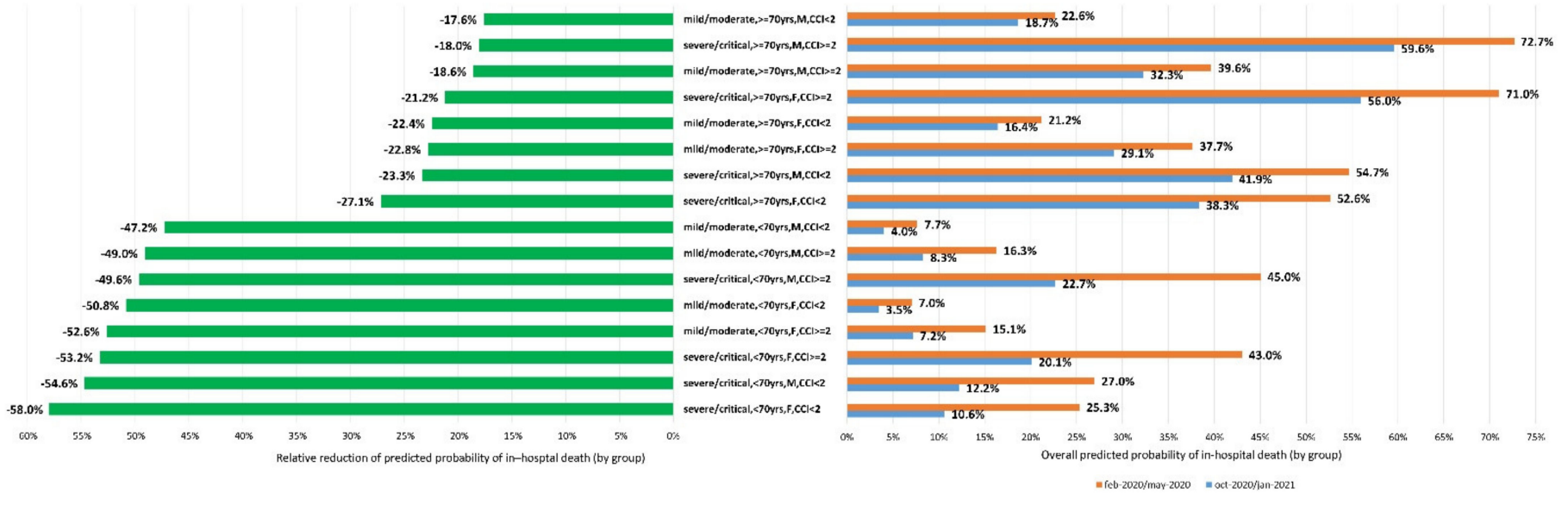

Figure 1. Marginal predictions of in-hospital death by sub-groups in first and second wave of epidemic (right panel) and relative reduction during the second wave (left panel). yrs = years; $\mathrm{F}=$ females; $\mathrm{M}=$ males; $\mathrm{CCI}=$ age unadjusted Charlson Comorbidity Index.

Given these findings, we analysed the probability of reduction of in-hospital death according to period separately, in patients younger and older than 70. After adjusting for all the variables listed in Table 2, patients younger than 70 showed $72 \%$ lower risk of death in the second wave (ASHR 0.28, 95\% CI: 0.16-0.48), while patients older than 70 showed only $26 \%$ of risk reduction (ASHR $0.74,95 \%$ CI: $0.56-0.99$ ).

\section{Discussion}

In our study population including over 1500 patients hospitalised for COVID-19 we demonstrated a significant decrease of the mortality rate between the first and second wave of the epidemics, albeit with people aged over 70 years benefiting to a lesser extent from this risk reduction. More specifically, the percentage of mortality reduction varied from $17.6 \%$ in the cluster of males, older than 70, with mild/moderate COVID-19 disease and Charlson index below 2 (in-hospital mortality rate from $22.6 \%$ in the first wave to $18.7 \%$ in the second one), to $58 \%$ in the cluster of females, younger than 70 , with severe/critical disease and Charlson index below 2 (in-hospital mortality rate from $25.9 \%$ in the first wave to $10.6 \%$ in the second one). It is noteworthy that a specific cluster of patients composed of men, above 70 , with critical/severe disease and Charlson $>2$ showed a very high in-hospital mortality rate in both calendar periods, with only slight reduction between the first and the second wave $(72.7 \%$ to $59.6 \%)$.

More specifically, the 1005 patients hospitalised in the second-wave period showed important differences compared to the 556 ones hospitalised in the first wave, the former being older and affected by more comorbidities and thus more likely candidates to a worse outcome. On the other hand, they showed the same severity of disease at admission and advantaged by more effective therapeutic support, such as remdesivir and corticosteroids, as the data from clinical trials became available meanwhile $[7,8]$.

Importantly, we excluded from the analyses all the patients dying in emergency room within $24 \mathrm{~h}$ but also the high number of patients who showed up at our emergency rooms but ended up not being hospitalised as they were suffering from a mild disease. The exclusion of this particular group may be among the explanatory factors behind the very high rates of in-hospital deaths in the study setting. Further, the small percentage $(19.5 \%, 48$ cases) of patients hospitalised in spite of not suffering from COVID-19-related pneumonia included mostly patients from long-term care facilities, with a high load of comorbidities. Nonetheless, the number of deaths in our hospital was also higher than the 
other cohorts in the Milan area, showing 23\% of fatality rate [9] but it should be emphasised than our population was the oldest one and with highest percentages of patients with at least two comorbidities ( $44 \%$ vs. $19 \%$ ).

Hoffmann and Wolf [10] showed very well that mortality rate relates to age, and that Italy, UK and France are those countries with the highest mortality rates and the oldest populations. Obviously, there might have been a selection bias in reporting cases and deaths by different countries, but the authors underline that any other speculations on different mortality rates across the countries, such as genetics, viral and socio-economic factors [11-13] should take into account age as a main driver of worse outcome in COVID-19.

Predictive factors of worse outcome other than age and comorbidities have been already addressed in our cohort [14] and are confirmed in this updated analysis with a more than doubled population. Being older, with high comorbidity load, high inflammatory markers at hospital admission, with severe/critical COVID-19 were confirmed to be associated with a worse outcome. Further, in this larger series, females were associated to a better outcome, as already reported by other authors $[15,16]$.

To underline, we excluded more recent periods from the analyses as possible variants, in detail B.1.617.2, the so called 'delta variant', known to be more contagious but probably less aggressive [17], might conditionate outcome, thus confounding our analyses.

Our study has several limitations: first, we did not collect the number of patients excluded as dying within $24 \mathrm{~h}$ from admission or those not requiring hospitalisation. Second, a number of variables that could be considered uncounted confounding factors are not adequately collected, most notably obesity [18]. This last variable seems to be not equally distributed among the two waves' patients, and might have affected the estimates of the risk of death. Third, even if we have adjusted our analyses for therapy intervention, the observational nature of our study cannot allow to completely adjust for as in a trial setting.

\section{Conclusions}

In conclusion, we observed a sharp reduction of in-hospital deaths according to the first two waves of SARS CoV-2 epidemics, but males aged above 70 showed less benefit of improved survival overtime and thus should be the focus of preventive campaigns and of vaccination programs.

\section{Patents}

All patients hospitalised for COVID-19 give their consent to treatment of data related to their illness by anonymous way.

Supplementary Materials: The following are available online at https:/ / www.mdpi.com/article/10 .3390/life11090979/s1. Supplemental Table S1. Demographic, clinical and laboratory characteristics and outcomes of 1683 patients according to the period of SARS cov-2 epidemics. Supplemental Table S2. Factors associated with in-hospital death in severe/critical subjects at admission by fitting a fine-Gray model.

Author Contributions: Conceptualisation, A.d.M.; data curation, F.B., D.T. (Daniele Tomasoni), C.F., R.C., D.B., G.M. (Giovanni Mulè), M.A. (Marina Allegrini), E.S., D.T. (Daniele Tesoro), G.T., D.M., M.A. (Matteo Augello), A.C. (Andrea Cona), T.B. (Tomaso Beringheli), N.G., M.S., B.V., F.M., S.P., L.B., A.C. (Alessandro Copes), O.V., C.T., A.D.B. and T.B. (Teresa Bini); formal analysis, A.T. and F.B.; investigation, D.T. (Daniele Tomasoni), C.F., R.C., D.B., G.M. (Giovanni Mulè), M.A. (Marina Allegrini), E.S., D.T. (Daniele Tesoro), D.M., M.A. (Matteo Augello), A.C. (Andrea Cona), T.B. (Tomaso Beringheli), N.G., M.S., B.V., F.M., S.P., L.B., A.C. (Alessandro Copes), L.G., O.V. and A.D.B.; methodology, A.T.; software, D.T. (Daniele Tomasoni) and G.T.; supervision, A.d.M. and G.M. (Giulia Marchetti); visualisation, L.G.; writing—original draft, A.d.M.; writing—review and editing, A.d.M., C.T., T.B. (Teresa Bini) and G.M. (Giulia Marchetti). All authors have read and agreed to the published version of the manuscript.

Funding: This research was founded by Fondazione di Comunità Milano, grant number FON_NAZ20ADARM_01. 
Institutional Review Board Statement: The study was approved by Ethic Committee Area 1, Milan (2020/ST/049 and 2020/ST/049_BIS, 11 March 2020).

Informed Consent Statement: Informed consent was obtained by the patients whenever possible.

Data Availability Statement: Upon request, the dataset can be available at our Institution.

Acknowledgments: We thank Laia Cirera and Nicola Tissi for proofreading the manuscript.

Conflicts of Interest: The authors declare no conflict of interest.

\section{References}

1. WHO. Covid19. Available online: www.who.int (accessed on 26 July 2021).

2. Wu, Z.; McGoogan, J.M. Characteristics of and important lessons from the Coronavirus disease 2019 (COVID 19) outbreak in China. Summary of a report of 72314 cases from the Chinese Center for Disease Control and Prevention. JAMA 2020, 323, 1239-1242. [CrossRef] [PubMed]

3. Williamson, E.J.; Walker, A.J.; Bhaskaran, K.; Bacon, S.; Bates, C.; Morton, C.E.; Curtis, H.J.; Mehrkar, A.; Evans, D.; Inglesby, P.; et al. Factors associated with COVID-19-related death using OpenSAFELY. Nature 2020, 584, 430-436. [CrossRef] [PubMed]

4. ECDC. COVID-19; Communicable disease threats report; Week 36, 5-11 September 2021. Available online: https:/ /www.ecdc.europa. eu/en/publications-data/communicable-disease-threats-report-5-11-september-week-36 (accessed on 15 September 2021).

5. Ministero Della Salute. Available online: https://www.salute.gov.it/portale/nuovocoronavirus/dettaglioNotizieNuovoCoronavirus. jsp?lingua=italiano\&menu=notizie $\& \mathrm{p}=$ dalministero\&id $=4648$ (accessed on 26 July 2021).

6. Charlson, M.E.; Pompei, P.; Ales, K.L.; MacKenzie, C.R. A new method of classifying prognostic comorbidity in longitudinal studies: Development and validation. J. Chron. Dis. 1987, 40, 373-383. [CrossRef]

7. Beigel, J.H.; Tomashek, K.M.; Dodd, L.E.; Mehta, A.K.; Zingman, B.S.; Kalil, A.C.; Hohmann, E.; Chu, H.Y.; Luetkemeyer, A.; Kline, S.; et al. Remdesivir for the treatment of Covid-19- Preliminary report. N. Engl. J. Med. 2020, 383, 1813-1836. [CrossRef] [PubMed]

8. The Recovery Collaborative Group. Dexamethasone in hospitalized patients with COVID-19. N. Engl. J. Med. 2021, 384, 693-704. [CrossRef] [PubMed]

9. Ciceri, F.; Castagna, A.; Rovere-Querini, P.; de Cobelli, F.; Ruggeri, A.; Galli, L.; Conte, C.; de Lorenzo, R.; Poli, A.; Ambrosio, A.; et al. Early predictors of clinical outcomes of COVID-19 outbreak in Milan, Italy. Clin. Immunol. 2020, 217, 108509. [CrossRef] [PubMed]

10. Hoffmann, C.; Wolf, E. Older age and country-specific case fatality rates of COVID-19 in Europe, USA and Canada. Infection 2021, 49, 111-118. [CrossRef] [PubMed]

11. Ghafouri-Fard, S.; Noroozi, R.; Vafaee, R.; Branicki, W.; Posjpiech, E.; Pyrc, K.; Łabaj, P.P.; Omrani, M.D.; Taheri, M.; Sanak, M. Effects of host genetic variations on response to, susceptibility and severity of respiratory infections. Biomed. Pharmacother. 2020, 128, 110296. [CrossRef] [PubMed]

12. Tang, X.; Wu, C.; Li, X.; Song, Y.; Yao, X.; Wu, X.; Duan, Y.; Zhang, H.; Wang, Y.; Qian, Z.; et al. On the origin and continuing evolution of SARS-CoV-2. Natl. Sci. Rev. 2020, 7, 1012-1023. [CrossRef]

13. Chakrabarti, S.S.; Kaur, U.; Banerjee, A.; Ganguly, U.; Banerjee, T.; Saha, S.; Parashar, G.; Prasad, S.; Chakrabarti, S.; Mittal, A.; et al. COVID-19 in India: Are biological and environmental factors helping to stem the incidence and severity? Aging Dis. 2020, 11,480-488. [CrossRef] [PubMed]

14. D'Arminio Monforte, A.; Tavelli, A.; Bai, F.; Tomasoni, D.; Falcinella, C.; Castoldi, R.; Barbanotti, D.; Mulè, G.; Allegrini, M.; Tesoro, D.; et al. The importance of patients' case-mix for the correct interpretation of the hospital fatality rate in COVID-19 disease. Int. J. Infect. Dis. 2020, 100, 67-74. [CrossRef] [PubMed]

15. Mussini, C.; Cozzi-Lepri, A.; Menozzi, M.; Meschiari, M.; Franceschini, E.; Rogati, C.; Cuomo, G.; Bedini, A.; Iadisernia, V.; Volpi, S.; et al. Better prognosis in females with severe COVID-19 pneumonia: Possible role of inflammation as potential mediator. Clin. Microbiol. Infect. 2021, 27, 1137-1144. [CrossRef] [PubMed]

16. Sharma, G.; Volgman, A.S.; Michos, E.D. Sex differences in mortality from COVID-19 pandemic: Are men vulnerable and women protected? JACC Case Rep. 2020, 2, 1407-1410. [CrossRef] [PubMed]

17. Reardon, S. How the Delta variant achieves its ultrafast spread. Nature, 21 July 2021. [CrossRef]

18. Simonnet, A.; Chetboun, M.; Poissy, J.; Raverdy, V.; Noulette, J.; Duhamel, A.; Labreuche, J.; Mathieu, D.; Pattou, F.; Jourdain, M.; et al. High prevalence of obesity in Severe Acute Respiratory Syndrome Coronavirus-2 (SARS-CoV-2) requiring Invasive Mechanical Ventilation. Obesity 2020, 28, 1195-1199. [CrossRef] [PubMed] 